\title{
CARACTERÍSTICAS QUÍMICAS DE SUBSTRATOS UTILIZADOS NA PRODUÇÃO DE MUDAS DE CACAUEIROS ${ }^{1}$
}

\author{
GEORGE ANDRADE SODRÉ2, JOSÉ EDUARDO CORÁ3 ${ }^{3}$ ISABEL CRISTINA SILVA FONTES LIMA BRANDÃO4, \\ MARIA HELENA DE CARVALHO FERNANDES SERÔDIO
}

\begin{abstract}
RESUMO - O objetivo deste trabalho foi realizar monitoramento químico de seis substratos utilizados na produção de mudas de cacaueiros. Os substratos receberam 10 volumes $(100 \mathrm{~mL}$, cada) de água destilada, e no lixiviado coletado foram determinados o pH, a condutividade elétrica (CE) e os teores de sódio $(\mathrm{Na})$ e potássio $(\mathrm{K})$ em solução. Os valores de $\mathrm{pH}$ variaram de 5,0 a 8,0 em Plantmax ${ }^{\circledR}$ e composto do tegumento da amêndoa do cacau (CTAC), respectivamente. Verificou-se que, a partir do primeiro volume lixiviado, a CE de todos os substratos foi reduzida. Foram encontradas correlações positivas para a CE em relação ao K e Na da fibra de coco (FC) (r=0,83 e 0,72 P<0,05) e CTAC (r=0,98 e 0,99 P <0,05). Os resultados indicam que a FC e o CTAC deveriam ser usados após pré-lavagens. Verificou-se que, embora a FC apresente na primeira lixiviação valor de CE superior aos demais substratos, o valor ideal da $2,0 \mathrm{dSm}^{-1}$ é alcançado na segunda lixiviação. Os teores de K e Na são um indicativo do valor da CE em FC e CTAC. Termos para indexação: Theobroma cacao L, método Pour Thru, lixiviados de substratos.
\end{abstract}

\section{CHEMICAL PROPERTIES OF SUBSTRATES USED IN CACAO PROPAGATION}

\begin{abstract}
The aim of this study was to monitor the chemical properties of six substrates used in cacao propagation. The substrates received ten volumes $(100 \mathrm{~mL}$ each) of distilled water and the resultant leached were analyzed for $\mathrm{pH}$, electrical conductivity (EC) and $\mathrm{Na}$ and $\mathrm{K}$ concentrations. The values of $\mathrm{pH}$ ranged from 5,0 to 8,0 in Plantmax ${ }^{\circledR}$ and composted cacao hulls (CTAC), respectively. It was confirmed that the CE of all substrates diminished after the first leaching volume. The results demonstrated that coconut fiber (FC) as well as CTAC should be used after an initial leaching pre-treatment. Yet while FC presented an initial EC greater than CTAC, the study showed that FC reaches the optimal value, $2.0 \mathrm{dSm}^{-1}$, with fewer leaching volumes. Moreover, a positive correlation was found between $\mathrm{EC}$ concentrations of $\mathrm{K}$ and $\mathrm{Na}$ from $\mathrm{FC}(\mathrm{r}=0.83$ and 0.72 ; $\mathrm{P}<0.05)$ and $\mathrm{CTAC}$ ( $r=0.98$ and $0.99 ; \mathrm{P}<0.05$ ), suggesting that the concentration of $\mathrm{K}$ and $\mathrm{Na}$ are adequate indicators of $\mathrm{EC}$ of these substrates.
\end{abstract}

Index Terms: Theobroma cacao L, Pour Thru method, leaching of substrates,

A produção de mudas de cacaueiros Theobroma cacao L, utilizando sementes ou estacas de ramos plagiotrópicos, não é uma técnica nova nessa cultura. Entretanto, com o surgimento de novos materias clonais, o uso da estaquia vem crescendo, destacando-se o Estado da Bahia, com produção de 6.641 .767 mudas entre julho de 1999 e julho de 2004 (Marrocos \& Sodré 2004). Uma das principais dificuldades para implementação dessa técnica tem sido o manejo dos substratos, isso devido à exigência de adaptação dos viveiros ao novo paradigma de substituição do solo por substratos para a produção comercial das mudas.

No manejo das características químicas de substratos, os principais atributos monitorados são o $\mathrm{pH}$, a capacidade de troca de cátions (CTC) e a salinidade (Kampf, 2000). A faixa de $\mathrm{pH}$ considerada ideal para os cultivos varia de acordo com o substrato, ambiente e cultura; por outro lado, a condutividade elétrica (CE) indica a concentração de sais ionizados na solução e auxilia na estimativa da salinidade do substrato.

Quando existem elementos com potencial para alteração da CE, como o sódio, é importante que se determine a concentração do elemento (Prieto Martinez \& Silva Filho, 2004). Esses autores enfatizam que os íons responsáveis pela elevação da CE podem ou não ser nutrientes de plantas.

As medidas de pH e CE, normalmente, são realizadas após extração aquosa nas proporções $1: 1,5 ; 1: 5$ ou extrato de saturação
(Bataglia \& Furlani, 2004). De acordo com esses autores, o uso de métodos não-destrutivos para monitorar quimicamente os substratos é ferramenta segura para tomada de decisão para a irrigação ou fertirrigação.

Este trabalho teve como objetivo avaliar características químicas de substratos utilizados na produção de mudas de cacaueiros.

O ensaio foi conduzido no laboratório de solos da Comissão Executiva do Plano da Lavoura Cacaueira da Ceplac, Ilhéus, Bahia. Foi utilizado o método Pour Thru (Cavins et al., 2000), com modificações. Esse método consiste em determinar o pH e CE no lixiviado obtido, uma hora após a irrigação de recipientes cultivados. Foram realizadas as seguintes modificações: uso de tubetes não cultivados e determinação de teores de $\mathrm{Na}$ e $\mathrm{K}$ no lixiviado.

Foram utilizados seis substratos, cujas principais características se encontram na Tabela 1. Para cada substrato, utilizaram-se tubetes plásticos de $288 \mathrm{~cm}^{3}$, que foram colocados sobre potes de vidro de 200 $\mathrm{mL}$. Os substratos receberam inicialmente $100 \mathrm{~mL}$ de água destilada, deixando escorrer por um período inicial de 14 horas. Para evitar a perda de material, no fundo dos tubetes, foram colados botões plásticos de 1,2 $\mathrm{cm}$ de diâmetro com quatro furos. Após o período inicial, o mesmo procedimento foi realizado nove vezes a intervalos de uma hora, recolhendo-se separadamente o volume lixiviado.

TABELA -1 Principais características de substratos utilizados na produção de mudas de cacaueiros.

\begin{tabular}{|c|c|c|}
\hline Nome & Principais características & $\begin{array}{l}\text { Densidade } \\
\text { úmida }^{(1)} \\
\mathrm{Kg}^{-3} \mathrm{~m}^{-3} *\end{array}$ \\
\hline Plantmax ${ }^{\circledR}$ eucalipto / Pmax & Produto comercial utilizado na silvicultura, custo mais elevado. & 622,38 \\
\hline Composto de tegumento da amêndoa do & Matéria-prima homogênea, disponível nas indústrias do sul da Bahia. & 295,88 \\
\hline Serragem / Ser & Baixo custo, material heterogêneo, alto teor de tanino. & 396,24 \\
\hline Fibra de coco / FC & $\begin{array}{l}\text { Subproduto da ind ústria de fibras aumenta a retenção de água, risco de } \\
\text { salinização. }\end{array}$ & 117,45 \\
\hline Casca de arroz carbonizada / CAC & Alto espaço de aeração, recomendada para misturas. & 187,70 \\
\hline Vermiculita / Ver & Alta retenção de água, granulometria variável. & 242,69 \\
\hline
\end{tabular}

${ }^{1}$ Método da auto compactação (Hoffmann, 1970, citado por Fermino, 1996).

${ }^{1}$ (Trabalho 137/2005). Recebido: 05/09/2005. Aceito para publicação: 09/12/2005.

${ }^{2}$ Pesquisador Ceplac/Cepec \& Professor Universidade Estadual de Santa Cruz - UESC, CP 07, CEP 45600-970, Itabuna-Ba; sodre@cepec.gov.br.

3 Professor Departamento de Solos e Adubos, Unesp, Jaboticabal-SP. cora@fcav.unesp.br.

${ }^{4}$ Bióloga, Ceplac/Cepec-Senup, CP 07, CEP 45600-970, Itabuna-BA. labsolo@cepec.gov.br.

${ }_{5}^{5}$ Pesquisadora Ceplac/Cepec- CP 07, CEP 45600-970, Itabuna-Ba. labsolo@cepec.gov.br. 


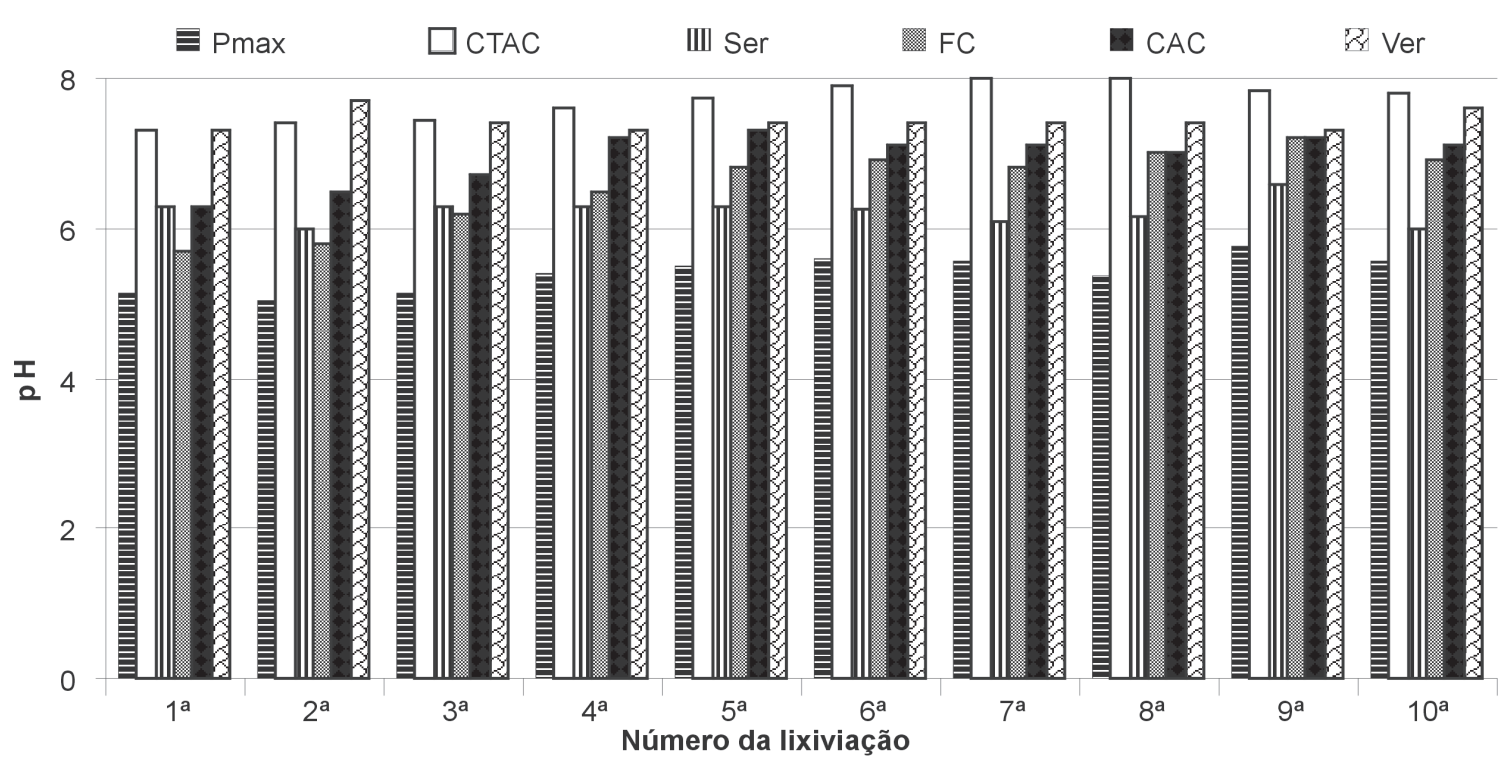

FIGURA 1- Valores médios de pH encontrados no lixiviado de substratos utilizados para produção de mudas de cacaueiros. Plantmax (Pmax); Composto do tegumento da amêndoa do cacau (CTAC); Serragem (SER); Fibra de coco (FC) Casca de arroz carbonizada (CAC) e Vermiculita (VER).
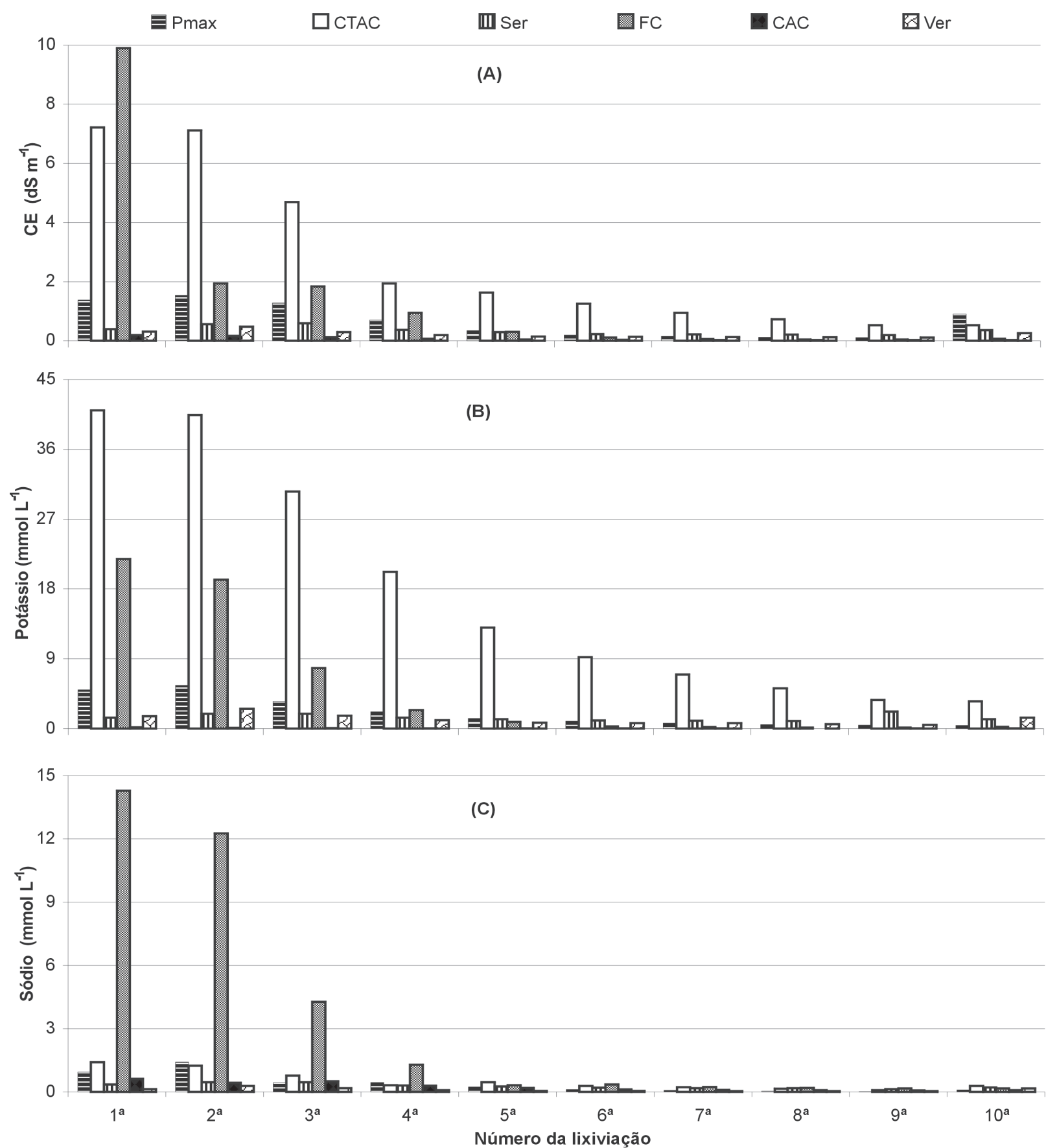

FIGURA 2- Valores médios de Condutividade elétrica CE, (A), Potássio (B) e Sódio (C) em solução, encontrados no lixiviado de substratos utilizados para produção de mudas de cacaueiros. Plantmax (Pmax); Composto do tegumento da amêndoa do cacau (CTAC); Serragem (SER); Fibra de coco (FC) Casca de arroz carbonizada (CAC) e Vermiculita (VER). 
Após cada lixiviação, determinaram-se $\mathrm{pH}, \mathrm{CE}$ e os teores em solução de Na e K, com leitura em fotômetro de chama. Foram utilizados três tubetes por repetição para cada substrato, e a análise estatística foi realizada com a correlação entre os valores médios da $\mathrm{CE}$ e teores de $\mathrm{Na}$ e K.

Os valores de $\mathrm{pH}$ variaram entre 5,0 e 8,0 para o Plantmax ${ }^{\circledR}$ (Pmax) e o composto do tegumento da amêndoa do cacau (CTAC), respectivamente. Os substratos apresentaram valores mínimos superiores a 5,0 nas dez lixiviações (Figura 2). Esse valor é considerado ideal, segundo Baumgarten (2002), para substratos comerciais. Por outro lado, os valores do CTAC e vermiculita (VER) foram superiores a 7,0 em todos os lixiviados. Essa característica (pH elevado), segundo Handreck \& Black (1999), pode limitar o uso desses substratos, pois está relacionada com risco de diminuição da disponibilidade de micronutrientes para as plantas.

Os valores de $\mathrm{CE}$ da casca de arroz carbonizada (CAC) e serragem (Ser) foram inferiores a $0,6 \mathrm{dSm}^{-1} \mathrm{em}_{\text {todos os lixiviados. Valores }}$ de CE em CAC e Ser inferiores a 0,09 e $0,7 \mathrm{dSm}^{-1}$ foram também encontrados por Guerrini \&Trigueiro (2004) e Fermino (1996), respectivamente. Esse último autor classificou esses substratos como de baixa salinidade ou que dificilmente poderiam alterar a CE da solução lixiviada. Por outro lado, a CE do primeiro lixiviado da fibra de coco (FC) e do CTAC foi 3,9 e 2,8 vezes superior a $2,0 \mathrm{dSm}^{-1}$, respectivamente, sendo este o limite superior de CE recomendado para mudas de cacaueiros, e 8,0 e 4,0 vezes superior ao do substrato comercial Pmax.

Verificou-se que, a partir do primeiro lixiviado, os valores de CE dos substratos foram reduzidos. Observa-se, na Figura 2, que, embora no primeiro lixiviado da FC a CE tenha sido superior aos demais substratos, o valor recomendado foi obtido já no segundo lixiviado.

Foram encontradas correlações positivas para a $\mathrm{CE}$ em relação ao $\mathrm{K}(\mathrm{r}=0,83$ e $0,98 \mathrm{P}<0,05)$ e ao $\mathrm{Na}(\mathrm{r}=0,72$ e $0,99 \mathrm{P}<0,05)$, nos lixiviados da FC e CTAC, respectivamente. Conforme verificado por Abreu et al. (2002), o aumento de CE devido à presença de íons alcança valores até onze vezes superiores em substratos adubados em relação à testemunha sem adubação. Correlações positivas entre concentração de íons em extrato aquoso de substratos e a CE também foram relatadas por Prieto Martinez \& Silva Filho (2004)

Os teores de $\mathrm{K}$ e $\mathrm{Na}$ encontrados nos lixiviados estão apresentados na Figura 2. Verificou-se que FC e CTAC apresentaram valores de 4,0 e 7,5 vezes superiores para $\mathrm{K}$ e 5,0 vezes para $\mathrm{Na}$, respectivamente, aos valores recomendados para produção de mudas de cacaueiros. Em termos práticos, estes resultados indicam que tanto a FC como o CTAC só deveriam ser usados após pré-lavagens.

A produção de mudas de cacaueiros com uso dos substratos Ser, Ver, CAC e Pmax, em princípio, pode ser utilizada sem necessidade de pré-lavagem, enquanto, FC e CTAC dependem desta prática. Os teores de $\mathrm{K}$ e $\mathrm{Na}$ foram indicativos do valor da CE dos substratos FC e CTAC.

\section{REFERÊNCIAS}

ABREU, M.F; ABREU, C. A; BATAGLIA, O.C. Uso da análise química na avaliação da qualidade de substratos e componentes. In: ENCONTRO NACIONALDE SUBSTRATOS PARA PLANTAS, 4., Campinas. Documentos... Campinas: IAC, 2002. p.19-28.

BATAGLIA, O.C.; FURLANI, P. R. Nutrição mineral e adubação para cultivos em substratos com atividade química. In: ENCONTRO NACIONAL DE SUBSTRATOS PARA PLANTAS,4., 2004. Viçosa, p.106-125.

BAUMGARTEN, A. Methods of chemical and physical evaluation of substrates for plants. In: In: ENCONTRO NACIONAL DE SUBSTRATOS PARA PLANTAS, 4., Campinas. Documentos... Campinas: IAC, 2002.p.7-15.

CAVINS, T. J.; GIBSON, J. L.; WHIPKER, B.E.; FONTENO, W.C. pH and ec meters - tool for substrate analysis. North Carolina State University, 2000. (Florex, 001).

FERMINO, M.H. Aproveitamento de resíduos industriais e agrícolas como alternativas de substratos hortícolas. 1996.90 f. Dissertação (Mestrado em Fitotecnia) -Universidade Federal do Rio Grande do Sul, Porto Alegre, 1996.

GUERRINI,I.A.; TRIGUEIRO, R.M. Atributos físicos e químicos de substratos compostados por biossólidos e casca de arroz carbonizada. Revista Brasileira de Ciência do Solo, Viçosa, v 28, p.1067-1076, 2004.

HANDRECK, K.; BLACK, N. Growing media for ornamental plants and turf. Sydney: University of New South Wales Press, 1999. 448p.

KÄMPF, A. N. Substrato. In: KÄMPF,A. N. (Coord.). Produção comercial de plantas ornamentais. Guaíba: Agropecuária, 2000.254p.

MARROCOS, P.C.L.; SODRÉ, G.A. Sistema de produção de mudas de cacaueiros. In: : ENCONTRO NACIONAL DE SUBSTRATOS PARA PLANTAS,4., 2004. Viçosa, p.283-311.

PRIETO MARTINEZ, H.E.; SILVA FILHO, J.B. Introdução ao cultivo hidropônico de plantas. 2.ed. Viçosa: UFV, 2004. 111p. 